\section{A CEREBRAL DIFFUSION WEIGHTED MRI STUDY TO ASSESS THE DIFFERENCE BETWEEN EMBOLIC EVENTS FOLLOWING TRANSCATHETER AORTIC VALVE IMPLANTATION (TAVI) AND SURGICAL AORTIC VALVE REPLACEMENT (SAVR)}

A U Uddin, ${ }^{1}$ T A F Fairbairn, ${ }^{1}$ I D Djoukhader, ${ }^{2}$ S C Currie, ${ }^{2}$ M M Motwani, ${ }^{1}$ A K Kidambi, ${ }^{1}$ A G Goddard, ${ }^{2}$ D B Blackman, ${ }^{3}$ S P Plein, ${ }^{1}$ J P G Greenwood ${ }^{1}$ ${ }^{1}$ Multidisciplinary Cardiovascular Research Centre \& The Division of Cardiovascular and Diabetes Research, LIGHT, Leeds University; ${ }^{2}$ Department of Neuroradiology, Leeds Teaching Hospitals NHS Trust, Leeds General Infirmary; ${ }^{3}$ Department of Cardiology, Leeds Teaching Hospitals NHS Trust, Leeds General Infirmary

doi:10.1136/heartjnl-2013-304019.144

Background Transcatheter Aortic Valve Implantation (TAVI) is used to treat symptomatic severe aortic stenosis in a non-surgical high risk population. The incidence of stroke and micro-infarction is higher in the TAVI population compared to surgical aortic valve replacement (SAVR), which may be due to various factors, including valve calcification and aortic atheroma. However, the natural history and clinical consequences of 'silent' micro-infarction is unknown.

Methods Cerebral imaging was conducted pre-TAVI/SAVR, at $<7$ days post-procedure and at 6 months. MRI scans were performed on a 1.5 T system (Intera, Philips or Avanto, Siemens) using a protocol of T2 weighted fast field echo, T2 turbo field echo and diffusion weighted imaging (DWI) (22 slices, $5 \mathrm{~mm}$ thick, $1 \mathrm{~mm}$ gap, FOV 350, RFOV 100). Three neuroradiologists independently analysed the scans and were blinded to all clinical details to avoid bias. New cerebral lesions were quantified ( $\mathrm{ml}$ of cerebral tissue) and the vascular territory described. Quantification of cerebral

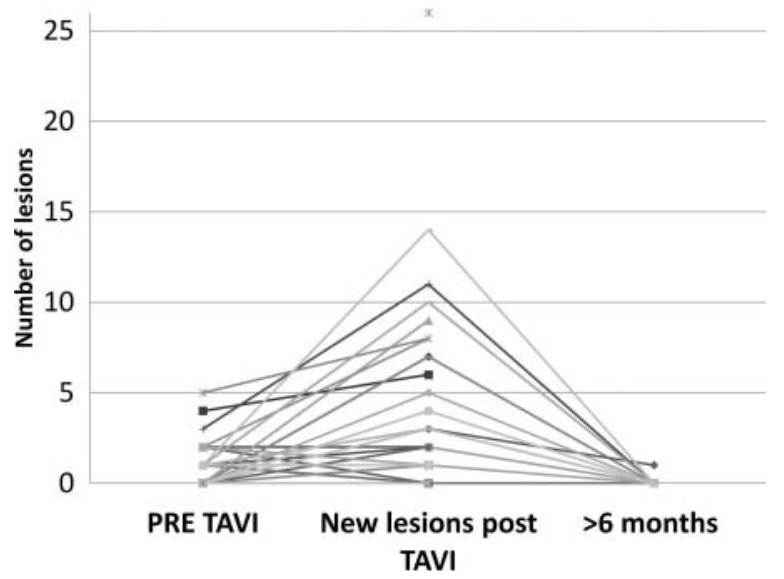

Figure 1 Number of lesions on brain diffusion weighted imaging (DWI) before and after TAVI in 45 patients and at 6 months in 17 patients.

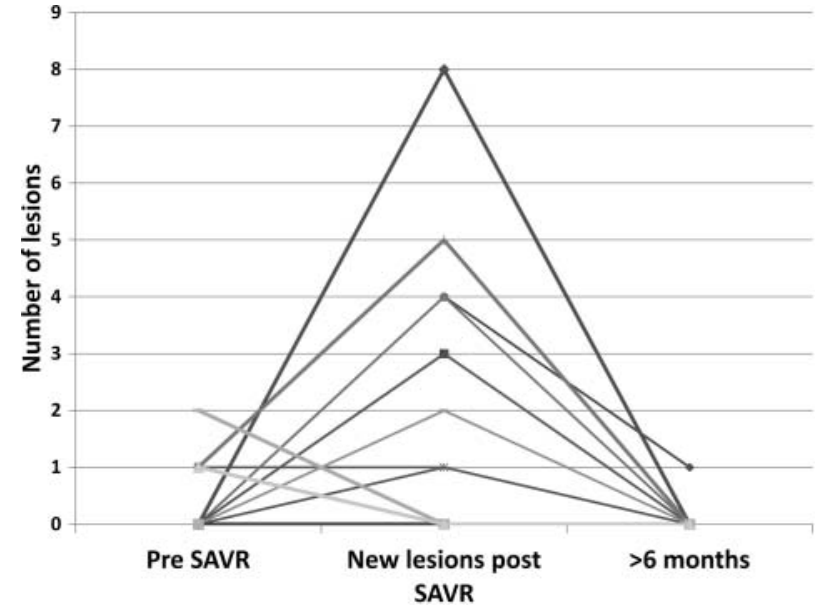

Figure 2 Number of lesions on brain diffusion weighted imaging (DWI) before and after surgical aortic valve replacement (SAVR) in 21 patients and at 6 months in 18 patients.

infarct lesion volume was performed by manual planimetry using post processing software (Omass 7.2 Medis, The Netherlands).

Results 45 TAVI and 21 SAVR patients were studied. Mean age for TAVI was $80 \pm 5.9$ years, for SAVR $69 \pm 8.8$ years: Logistic EuroSCORE for TAVI was $19.1 \pm 13$, for SAVR 7.1 \pm 2.7 . In the TAVI patients $82 \%$ had new embolic lesions on DWI (figure 1), compared to $47.6 \%$ of the SAVR group. Two TAVI patients had a clinical stroke compared to none in the SAVR group. There was a higher cerebral infarct lesion volume in TAVI group compared to SAVR group $(1.74 \pm 2.8 \mathrm{ml}$ vs $0.41 \pm 0.48 \mathrm{ml} \mathrm{p}=0.01)$. At 6 months 17 TAVI patients (11 were unable to attend, 10 had died, 7 had a pacemaker) and 18 SAVR (1 died and 1 was unable to attend) were re-scanned. Only 1 TAVI and 1 SAVR patient had a new subclinical micro-infarct. All previously detected micro-infarcts had completely resolved.

Conclusions There is a significantly greater number of new microembolic events after TAVI compared to SAVR. However all of these lesions appear to completely resolve on DWI MRI by 6 months.

Funding SP is funded by a British Heart Foundation fellowship (FS/ 10/62/28409). SP and JPG receive an educational research grant from Philips Healthcare. 